\title{
Frecuencia de uso de palabras gramaticales en textos académicos:
}

Comparación de un corpus de aprendices de ELE con tres corpus de referencia

\author{
María del Pilar VALVERDE IBÁÑEZ
}

Palabras clave: palabras gramaticales, interlengua,

español como lengua extranjera, lingüística de corpus

\section{Introducción}

Los textos escritos por aprendices de español como lengua extranjera se diferencian de los textos escritos por hablantes de español como primera lengua no solo por el mayor número de errores gramaticales o léxicos que contienen sino también por el uso de distintas estrategias de producción (secuencias textuales, cohesión, atenuaciones, marcas de quien escribe, etc.) (Gallardo 2005). Ello se refleja en la mayor o menor frecuencia de algunas palabras y construcciones y hace que, sobre todo en los niveles intermedio y superior, a menudo los aprendices escriban textos que, a pesar de ser correctos, no "suenan nativos".

En este trabajo, a partir del análisis de la frecuencia de uso de palabras gramaticales (determinantes, pronombres, conjunciones y preposiciones) en un corpus de monografías universitarias (resúmenes de tesinas de graduación) escritas por estudiantes japoneses con un nivel intermedio de español, nos proponemos revelar algunas insuficiencias de este tipo de textos respecto al modelo nativo de referencia.

Hemos escogido las palabras gramaticales y no otro tipo de palabras por varios motivos. En primer lugar, porque la presencia de palabras gramaticales nos informa de manera indirecta sobre algunas propiedades de los textos como el uso de mecanismos de cohesión y la presencia de cláusulas coordinadas y 
subordinadas, entre otros. En segundo lugar, mientras que los profesores de lengua suelen llamar la atención sobre la necesidad de evitar la repetición excesiva de palabras léxicas (con el uso de sinónimos, la elisión, etc.,) la frecuencia de las palabras gramaticales pasa más desapercibida, es más difícil detectar los patrones de uso que se desvían de la norma porque se trata de palabras que por su naturaleza se repiten necesariamente en los textos.

Los profesores de español tenemos algunas intuiciones sobre qué palabras gramaticales usan los aprendices de ELE con una frecuencia bastante superior o inferior a la del modelo nativo. Por ejemplo, es probable que los aprendices abusen de la conjunción porque y usen muy poco el determinante relativo cuyo. Sin embargo, tenemos pocas certezas: ¿en qué medida usan mucho o poco estas palabras? El uso de corpus nos va a permitir encontrar y cuantificar de forma más precisa esas intuiciones.

A continuación describimos los corpus usados en el estudio (2) y el método de extracción de palabras usadas con una frecuencia mayor o menor de lo esperable (3), comentamos con detalle la lista de palabras resultante (4) y extraemos algunas conclusiones (5).

\section{Descripción de los corpus}

El corpus objeto de estudio es un corpus de aprendices de ELE (2.1) compuesto por resúmenes de tesinas de graduación de estudiantes universitarios japoneses de la especialidad de español. Este corpus de aprendices, de 64.129 palabras, es comparado con tres corpus académicos que consideramos como corpus de referencia ${ }^{1)}$ (2.2), de características y tamaño variables: el corpus PAAU (350.000 palabras), formado por pruebas de acceso a la universidad realizadas en España, el corpus UNAM (230.000 palabras), compuesto por textos de estudiantes de la Universidad Nacional Autónoma de México, y un corpus creado a propósito para esta investigación, que denominamos UNED, compuesto por tesis de doctorado de la Universidad Nacional de Educación a Distancia (2,5 millones de palabras).

\subsection{Corpus de aprendices de ELE}

El corpus de aprendices de ELE está formado por 29 resúmenes de tesinas 
de graduación escritas por estudiantes japoneses de la especialidad de español de la Universidad Provincial de Aichi (APU), en Japón. Cada resumen tiene una extensión media de 2.211 palabras (no se han incluido la portada, el índice, la bibliografía y los apéndices de los resúmenes) y el tamaño del corpus es de aproximadamente 64.000 palabras. Los resúmenes fueron escritos entre 2013 y 2014, cuando los estudiantes se encontraban en el último año de carrera, y tenían un nivel de español intermedio (niveles B1 y B2 del Marco Común Europeo de Referencia para las lenguas).

En esta universidad, para poder graduarse, los estudiantes de último curso de la especialidad de español tienen que escribir un resumen en español de su tesina de graduación y superar también una entrevista en español sobre el tema de su tesina. Para ello, durante el último curso reciben una clase a la semana sobre español académico. En el primer semestre, se trabaja con distintos tipos de textos académicos (el resumen, la descripción, la narración y la argumentación), mientras que el segundo semestre está enfocado a la adquisición de las destrezas necesarias para la presentación escrita y oral de la tesina en español (el formato de la tesina, la introducción de un trabajo académico, la cohesión textual, el léxico, el uso de conectores, etc.).

Los resúmenes, que tratan temas relacionados con el mundo hispánico (cultura, lengua, política, literatura, etc.), fueron escritos con material de apoyo (diccionarios, gramática, artículos de investigación en español sobre su tema de investigación, etc.), comentados por el profesor nativo en cuanto a presencia de errores gramaticales o de contenido y fragmentos de difícil comprensión (sin corrección por parte del profesor), y corregidos por los propios estudiantes. Se trata, por lo tanto, de textos que contienen algunos errores, pero menos de los que encontraríamos en textos escritos sin material de apoyo y sin revisión.

\subsection{Corpus académicos de referencia}

El corpus PAAU (Battaner y Torner, 2005), de 350.000 palabras, está formado por aproximadamente 700 pruebas de acceso a la universidad celebradas en junio de 1992 en España (Barcelona, Madrid, Murcia, Oviedo, Salamanca y Sevilla). Contiene exámenes Comunes (Comentario de Texto, 
Filosofía), del área de Humanidades (Historia Contemporánea, Historia del Arte, Literatura) y del área de Ciencias (Biología, Matemáticas, Física, Geografía, Química).

El corpus CLAE (2009) es una recopilación de textos académicos de distinto tipo (reporte, reseña, ensayo, resumen, etc.) escritos por estudiantes universitarios en diferentes disciplinas (Historia, Geografía, Lengua y Literatura), en la Universidad Nacional Autónoma de México (UNAM) y la Universidad de California, Davis. Para esta investigación, empleamos solamente los textos producidos por nativos monolingües, procedentes de la Universidad Nacional Autónoma de México. Esta parte del CLAE, que en este artículo denominamos corpus UNAM, tiene un tamaño de 230.000 palabras.

Ante la necesidad de un corpus de español académico de gran tamaño que se adaptara a nuestro propósito, construimos un corpus con 15 tesis de doctorado extraídas de la web de la Biblioteca de la Universidad Nacional de Educación de Distancia (UNED) ${ }^{2}$. Seleccionamos tesis de las áreas de conocimiento a las que pertenecen las tesinas del corpus de aprendices: cinco tesis del área de Filología, cinco de Política y Economía y cinco de Cultura y Filosofía. El corpus resultante contiene aproximadamente 2,5 millones de palabras, tras descartar el material introductorio (portada e índices), bibliografía, apéndices y encabezados y números de página.

\section{Método de extracción de palabras usadas en mayor o menor medida}

Para la extracción de las palabras usadas en mayor o menor medida en el corpus de aprendices de ELE con respecto a los corpus de referencia, en primer lugar realizamos un análisis morfológico automático de todos los textos (3.1), que consiste en asignar un lema y una categoría gramatical a cada palabra. En segundo lugar, comparamos la frecuencia de cada determinante, pronombre, conjunción y preposición en el corpus de aprendices, en su forma de diccionario, con su frecuencia en los corpus de referencia. Con ello, obtenemos una lista de palabras clave (3.2), esto es, palabras usadas con una frecuencia inusualmente alta o baja en el corpus de aprendices respecto a los corpus de referencia. 


\subsection{Análisis morfológico}

Para poder extraer las frecuencias de uso de las palabras gramaticales asignamos primeramente un lema y categoría gramatical a cada palabra de los corpus. No podemos simplemente comparar las palabras flexionadas of formas, tal como se encuentran en el texto, por dos motivos. En primer lugar, algunas de las palabras gramaticales objeto de estudio (los determinantes y pronombres) admiten flexión de género y número, pero no nos interesa estudiar cada forma por separado (este, esta, estos, estas) sino agruparlas bajo una única forma de diccionario o lema (este). En segundo lugar, una única forma puede pertenecer a más de una categoría gramatical (por ejemplo este puede ser un determinante o pronombre, bajo puede ser una preposición, un sustantivo o un adjetivo, etc.), y por lo tanto para poder diferenciar cada caso es necesario asignar también una categoría gramatical a cada palabra.

Etiquetar cada palabra de los corpus de forma manual con un lema y una categoría gramatical sería una tarea tediosa y costosa si se hace de forma manual. Afortunadamente, disponemos de herramientas automáticas (analizadores morfológicos), como el analizador FreeLing (Padró y Stanislovsky, 2012), que a pesar de haber sido diseñado para tratar texto nativo pueden ser usado en textos escritos por aprendices de nivel intermedio con una precisión razonable (Valverde, 2011).

Para cada palabra, el analizador nos proporciona el lema, la categoría gramatical y otra información morfológica (género, número, tiempo verbal, modo, persona, etc.), como muestra el Cuadro 1. Cada línea corresponde a una palabra, y las columnas contienen la forma, el lema y la etiqueta. Esta última nos informa sobre la categoría gramatical $(\mathrm{RG}=$ adverbio, $\mathrm{SPS} 00=$ preposición, $\mathrm{NP}=$ nombre propio, $\mathrm{CC}=$ conjunción coordinada, $\mathrm{P}=$ pronombre, $\mathrm{V}=$ verbo, etc.) y otras propiedades morfológicas ${ }^{33}$.

\subsection{Análisis de palabras clave}

Una vez los corpus han sido etiquetados con información morfológica, comparamos la frecuencia de las palabras gramaticales en el corpus de aprendices con su frecuencia en los corpus de referencia. Con ello, extraemos la lista de palabras que son usadas en mayor o menor medida, denominadas 
palabras clave.

El método de extracción y análisis de listas de palabras clave ha sido propuesto para investigar las diferencias entre pares de corpus o variedades de lengua (Kilgarrif 2012, 2013). En esta área de investigación, encontramos estudios principalmente para el inglés, sobre las diferencias entre el registro escrito y oral (Biber 1999, 2006), o sobre las características particulares del lenguaje académico de los aprendices de inglés como lengua extranjera (Paquot 2010). En español, por el momento no existen estudios exhaustivos de este tipo debido a la falta de corpus representativos

Para consultar nuestros corpus y extraer la lista de palabras clave usamos la herramienta Sketch Engine (Kilgarrif et al. 2014), y su función Keywords. La Figura 1 muestra las condiciones de búsqueda para obtener la lista de determinantes que se usan con una frecuencia inusualmente alta en el corpus de aprendices respecto al corpus UNED: queremos extraer lemas con una determinada categoría gramatical (el elemento de búsqueda es "lempos"), delimitamos la búsqueda a los determinantes (con la expresión regular ${ }^{5)}$ *-x), tomamos como corpus de referencia el corpus de tesis de la UNED y preferimos extraer palabras con una frecuencia alta (ajustamos el parámetro a 100)

Con estas condiciones, la herramienta calcula, para cada combinación de lema-categoría gramatical (por ejemplo este como determinante, este como

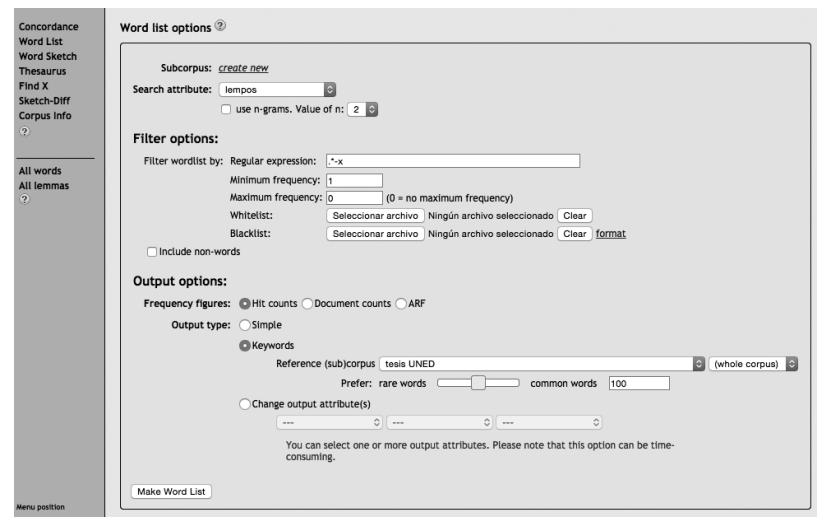

Figura 1: Interfaz de búsqueda de palabras clave en Sketchengine. 
pronombre, bajo como preposición, etc.), un puntaje (Keyness score) que nos informa sobre cuánto más frecuente es una palabra en el corpus objeto de estudio con respecto al corpus de referencia, con la fórmula siguiente (Kilgarrif 2009), donde fpmaprendices es la frecuencia por millón de una combinación de lema-categoría gramatical en el corpus de aprendices y fpmreferencia es la frecuencia por millón de esa misma combinación en el corpus de referencia. El valor de n (que en nuestro caso es 100) es un parámetro de suavizado, que decide el usuario dependiendo de su interés en las palabras más o menos frecuentes.

$$
\text { Keyness score }=\frac{f p m \text { aprendices }+n}{\text { fpm referencia }+n}
$$

Por ejemplo, el determinante mисho aparece 146 veces en el corpus de aprendices (esto es, 1979,9 veces por millón de palabras) y 1.261 veces en el corpus de tesis de UNED (esto es, 421,3 veces por millón de palabras). Por lo tanto, de acuerdo con la fórmula siguiente, este determinante es 4,6 veces más frecuente en el corpus de aprendices que en el corpus de nativos.

$$
\text { Keyness score }=\frac{\text { fpm aprendices }+n}{\text { fpm nativos }+n}=\frac{1979,9+100}{421,3+100}=4,6
$$

El resultado del análisis es una tabla con los lemas ordenados de mayor a menor puntaje, su frecuencia absoluta y frecuencia por millón en los dos corpus. En los Cuadros 2, 3 y 4 podemos ver el resultado de la consulta anterior, sobre los determinantes (la equis tras el lema indica que se trata de determinantes), en los tres corpus de referencia).

Para obtener la lista de lemas que se usan con una frecuencia inusualmente baja en el corpus de aprendices, modificamos las condiciones de la consulta de la Figura 1 e intercambiamos el corpus objeto de estudio y el corpus de referencia. El resultado se muestra en los Cuadros 5, 6 y 7 para los determinantes. 


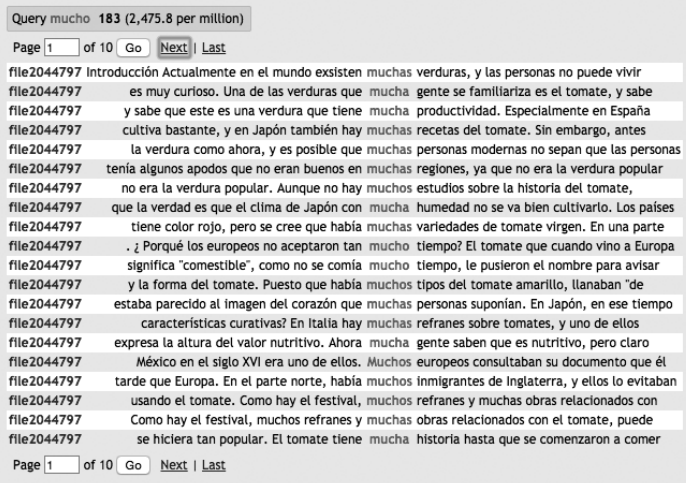

Figura 2: Concordancias del determinante mucho en el corpus de aprendices.

\section{Resultados}

Tras la extracción de la lista de palabras clave, examinamos en detalle los contextos de aparición de esas palabras por medio de concordancias como las de la Figura 2, para estudiar con más detalle sus contextos de aparición.

A continuación comentamos los resultados extraídos con ayuda de algunos ejemplos extraídos de los corpus. ${ }^{7)}$ Hemos seleccionado los casos más extremos de la distribución, que se apartan más del modelo de referencia. El lector interesado puede consultar la frecuencia concreta de cada lema en los cuadros adjuntos al final del artículo.

4.1. Palabras con una frecuencia inusualmente alta:

4.1.1. El determinante mucho

4.1.2. El pronombre ese

4.1.3. El pronombre ellos

4.1.4. La conjunción porque

4.1.5. La preposición según

4.2. Palabras con una frecuencia inusualmente baja:

4.2.1. El determinante aquel

4.2.2. El determinante nuestro

4.2.3. El pronombre ello 


\subsubsection{Los pronombres relativos}

4.2.5. Los pronombres de primera persona del plural nos, nosotros, nuestro

4.2.6. La conjunción pues

4.2.7. Las preposiciones tras, ante y hacia

\subsection{Palabras con una frecuencia inusualmente alta}

\subsubsection{El determinante mucho}

En el corpus de aprendices el determinante mucho se usa con una frecuencia extraordinariamente alta (Cuadros 2-4), no solo en las colocaciones habituales (mucho + tiempo, casos, veces, años, personas, gente, etc.) sino también como cuantificador indefinido preferido, que en ocasiones (1-4) podría ser sustituido por un adjetivo antepuesto de cantidad, dependiendo del sustantivo que antecede (diversos países, enormes gastos, grandes diferencias, larga historia, etc).

(1) Por otra parte, después de desplomarse el Imperio Otomano, Turquía y muchos países balcánicos fueron fundados, y en esos países se tomaron la política de asimilación y estaba prohibido usar lenguas salvo de la lengua oficial de ese país. (APU)

(2) El año siguiente España concretó el tratado de Basel porque sufría por los muchos gastos de guerra. (APU)

(3) Hay muchas diferencias de la enseñanza entre la zona repúblicana y la zona nacional. (APU)

(4) El tomate tiene mucha historia hasta que se comenzaron a comer. (APU)

\subsubsection{El pronombre ese}

Los aprendices de ELE usan los pronombres ese (Cuadros 8-10) principalmente para expresar causa, en la expresión por eso (5), que en ocasiones podría sustituirse por otra equivalente (por ello, por ese motivo, por esa causa, etc). 
(5) Las acllas eran mujeres escogidas para servir a el Inca o a el Dios Sol, por eso tenían que ser bellas. (APU)

En cambio, en los corpus de referencia se usa principalmente para aludir a algo que se ha mencionado antes y así dar cohesión al texto (6-8):

(6) Este hombre culto tiene un modo de ser que consiste en, utilizando las expresiones de D. Mundo178, estar-permanentemente-estimulado, o lo que es lo mismo, la clave de la cultura es que el sujeto sea un serentre-tenido, cuando su espíritu se estanca queda alienado y precisamente esa es la clave de la cultura moderna, una cultura que ahoga y no permite que el hombre vaya más allá de lo que ésta le ofrece (UNED).

(7) Los espacios porosos del suelo, por ejemplo, son ocupados por agua y por aire y eso influye de manera directa en la Densidad Real del suelo en estudio. (UNAM)

(8) La televisión no dedica espacios al arte solo emite series americanas de poco sentido, esa es una causa de su mala situación (PAAU)

\subsubsection{El pronombre ellos}

En el corpus de aprendices se usa el pronombre ellos (Cuadros 8-10) casi exclusivamente como sujeto de persona. Se trata de un uso redundante ya que a menudo el pronombre podría ser elidido (9).

(9) Una de las características más interesantes de las acllas es el respeto a la virginidad. Ellas tenían que ser vírgenes porque era posible que fueran esposas del Inca o del Dios Sol que eran existencias muy sagradas. (APU)

En los corpus de referencia, en cambio, se emplea el pronombre ellos principalmente para referirse a algo que se ha mencionado antes (10-12), actuando de nuevo como mecanismo de cohesión. 
(10) En conclusión, la propuesta de Lévi-Strauss de concepto de cultura tiene varios planos. Cuando habla de cultura puede referirse a cualquiera de ellos según el contexto de su obra, lo que hace de la cultura un agente complejo en el conjunto de su pensamiento. (UNED)

(11) La principal diferencia que hay entre una célula vegetal y una animal es la de los cloroplastos, éstos aparecen en la célula vegetal, y gracias a ellos se les permite a la planta que realice la fotosintesis. (PAAU)

(12) Un concepto básico para conocer la importancia que tiene para estas tribus el reconocimiento como sociedad específica con sus particularidades es la existencia en cada una de ellas de un "tótem" tribal (palabra extraída del vocabulario de los indios algonquinos de América del Norte, que sirve para designar el objeto que sirve de patrón del clan), un signo que representa a su espíritu protector y un símbolo que sirve para unificar a todo el pueblo. (UNAM)

\subsubsection{La conjunción porque}

Nuestros aprendices de ELE usan la conjunción causal porque tres veces más que los autores nativos de los corpus de referencia (Cuadros 14-16), a pesar de que existen otros mecanismos para expresar la causa.

Podrían usarse otras conjunciones causales (ya que, debido a que, etc.) (13) o, sobre todo cuando el sujeto de las dos cláusulas es el mismo $(14,15)$, la preposición por seguida de un sustantivo o infinitivo (por el desconocimiento, por los gastos):

(13) Sin embargo, la política no afectó al porcentaje de la población escolar. Al fin, el partido no pude influenciar mucho a la situación de los jóvenes. Eso es porque la familia pobre quiere que los hijos trabajen y es difícil que los jóvenes sigan trabajando después de la educación primaria. (APU)

(14) En consecuencia, los españoles predicaron pero los indios no pudieron comprender bien porque los españoles no saben la esencial 
cultura indígena. (APU)

(15) El año siguiente España concretó el tratado de Basel porque sufría por los muchos gastos de guerra. (APU)

En otros casos (16-18), se podría sustituir la conjunción causal por una conjunción o locución preposicional de finalidad como para que:

(16) Darwin dice que la cruz entre las especies diferentes es importante porque nace descendiente fuerte y diverso. (APU)

(17) En cuanto avisó la marcha de Versalles, Floridablanca disolvió de prisa las Cortes el 17 de octubre en 1789 porque temió que las Cortes se hicieran como la Asamblea Nacional. (APU)

(18) Así que existe la posibilidad de que los patrones no los afilien al seguro porque quieren el bajo coste. (APU)

En ocasiones porque se usa erróneamente en lugar de por (los) que (equivalente a por el cual, por la cual, por los cuales o por las cuales.) (19):

(19) Creo que uno de los factores porque los estudiantes japoneses son pasivos la participación a la conversación es la vacilación por diferencia del nivel de conocimientos. (APU)

Por último, los aprendices también usan porque para explicar las razones de sus decisiones u opiniones de manera directa $(20,21)$. Tratándose de textos académicos, sería preferible reorganizar la frase y expresar sus razones de manera más indirecta (tal como, que, etc.).

(20) En mi opinión, las reacciones del profesor tienen relación con la participación en la conversación de los estudiantes porque en una encuesta que había hecho en la Universidad Provincial de Aichi sobre las clases, había habido el comentario de "me da miedo de la reacción del profesor cuando cometo errores". (APU)

(21) Para investigar la política social del partido, utilizo las tesis de 
Verónica Amarante, Andrea Vigorito y otras porque investigaron PANES detalladamente. (APU)

\subsubsection{La preposición según}

Los aprendices usan con una frecuencia más alta de la esperada la preposición según (Cuadros 20-22), sobre todo para informar sobre el origen de una información $(22,23)$.

(22) Según Stingl(1986), Pachacti,el noveno gobernante del estado Inca, estableció una ley del incesto entre la casa real con el fin de que aumentase la pureza de sangre. (APU)

(23) En la educación primaria, la lengua enseñada es la lengua oficial según la idea de la democracia, que la lengua debe ser compartida igualmente entre la gente. (APU)

Con esta función, en los corpus de referencia encontramos no solo la preposición según sino también la locución preposicional de acuerdo con (que aparece solo una vez en el corpus de aprendices) (24).

(24) De acuerdo con las Ordenanzas, el Hospital de Malinas funcionó como centro de enseñanza para personal auxiliar. (UNED)

\subsection{Palabras con una frecuencia inusualmente baja}

\subsubsection{El determinante aquel}

En los corpus de referencia se usa el determinante aquel (Cuadros 5-7) sobre todo para hacer referencia anafórica (25), es decir, para remitir a otros elementos del contexto (aquel + momento, época, tiempo, persona, etc.), un mecanismo que los aprendices usan con poca frecuencia.

(25) En el prólogo de esa revista, Georg Mellis afirmaba que en aquel momento no existía un sistema prevaleciente de filosofía, lo que exigía tener que prestar atención a temas concretos que se iban proponiendo. (UNED) 
También se puede usar aquel como alternativa formal del artículo determinado, sobre todo cuando precede cláusulas relativas, con antecedente (26) o sin él (27).

(26) Las competencias básicas son aquellas expresiones didácticas en que se sintetiza el ser, saber y el hacer del profesional universitario y que sustentan su desempeño ante el objeto de la profesión, están en la base de las competencias profesionales. (UNAM)

(27) Los aminoácidos esenciales son aquellos que pueden sintetizar todos los individuos de una especie. (PAAU)

\subsubsection{El determinante nuestro}

La alta frecuencia del determinante nuestro (Cuadros 5-7) en los corpus de referencia revela que la primera persona del plural se usa frecuentemente en textos académicos para referirse a entidades relacionadas con la persona que escribe (nuestro + país, opinión, punto de vista, tiempo, investigación, estudio, etc.) (28).

(28) En nuestra opinión, el método de Bergson es problemático, ya que parte de la división entre el cuerpo y el espíritu como unidades aparentemente separadas para investigar los vínculos que los unen. (UNED)

La baja frecuencia de la primera persona del plural en el corpus de aprendices puede deberse a una ultracorrección. En clase se les insiste en que deben evitar la primera persona del singular en los textos académicos expositivos, y por extensión tienden a evitar también el uso de la primera persona del plural.

\subsubsection{El pronombre ello}

En cuanto al pronombre ello (Cuadros 11-13), apenas hay cinco casos en el corpus de aprendices, en los que forma parte de locuciones causales o concesivas como por ello y a pesar de ello (29). 
(29) Por gran diferencia de la población, la ayuda del portugués en el parte de gobierno, médico y buscar el trabajo es considerable. Por ello, quizas los brasileños que no saben japones en Toyokawa piden el parte de médico y buscar el trabajo en Toyohashi. (APU)

En cambio, los autores de los corpus de referencia usan este pronombre con mayor frecuencia, no en estructuras fijas sino, de nuevo, para referirse a algo que se ha mencionado antes, lo que da cohesión al texto (30-32).

(30) La crisis afecta a todos los países pero los medios no son iguales y en parte depende de ello la mejora. (PAAU)

(31) Muchos factores personales pueden influir en la decisión de consumir drogas, sin que podamos reducirlo exclusivamente a ello, éstas pueden ser vistas como una vía de escape a los problemas cotidianos; algunas personas las usan como medio para compensar frustración, soledad, baja autoestima o problemas afectivos, en efecto, bajo el efecto de las drogas la persona experimenta un estado de euforia que le hace olvidar los problemas o las limitaciones que tenga. (UNAM)

(32) El ser humano está destinado a participar de la ciencia, la moral y el arte, ello le hace verdaderamente humano. (UNED)

\subsubsection{Los pronombres relativos}

Como es esperable, los aprendices de ELE usan con una frecuencia inusualmente baja los pronombres relativos (quién, cuyo, donde, dónde, cual, aquel + que, a lo que, en que, etc) (Cuadros 11-13), que hacen la oración más compleja. Por ejemplo, en el ejemplo 33 el aprendiz ha escrito dos frases independientes que podrían unirse con en el que.

(33) Entre los hispanos también estudiar español tiene imagen de pobreza. Entonces, nació la tercera programa, "inmersión bidireccional". En este curso participan no solo los hispanos sino los mayorías también. (APU) 


\subsubsection{Los pronombres de primera persona del plural nos, nosotros, nuestro}

Los pronombres de primera persona del plural (nos, nosotros, nuestro) (Cuadros 11-13), como en el caso del determinante nuestro (4.2.2), son poco usados por los aprendices de ELE (34-35).

(34) A continuación, hay investigación por Núñez y Guerrero(2002) y ellos nos presentan las cuatro especies de los insultos políticos. (APU)

(35) Aunque sabemos que es fundamental respetar la lengua para nosotros, hemos cerrado los ojos a esa verdad dentro de los derechos humanos. (APU)

En los corpus de referencia se usan con más frecuencia, ya sea para referirse al ser humano en general $(36,37)$, o al autor del texto (38).

(36) Por un lado, la cultura muestra cómo debemos comportarnos en el día a día de nuestra vida, nos ofrece razones para actuar de una manera y no de otra. (UNED)

(37) Las cosas son para Ortega orden, ley, prescripción superior a nosotros, de ahí que pueda decir que las verdaderas realidades, las verdaderas « cosas », son suprapersonales o universales. (UNED)

(38) Por otra parte, los impactos y las consecuencias de un trasvase intercuencas siempre suelen ser más importantes que en el caso de trasvases intracuencas, por lo que los análisis realizados en este trabajo son perfectamente válidos para el nuestro. (UNED)

\subsubsection{La conjunción pues}

En contrapartida a la alta frecuencia de porque, los aprendices usan con poca frecuencia la conjunción causal pues (Cuadros 17-19). En los corpus de referencia se usa pues para expresar causa (39), en la expresión consecutiva así pues (40) o después del verbo se trata (41), entre otros. 
(39) El problema de las drogas dejo de ser un problema de salud, para convertirse en un problema de gran escala pues abarca múltiples sectores de la sociedad en todo su conjunto. (UNAM)

(40) La obra se corresponde con la posguerra de la guerra civil española. Así pues es característico el malestar social que se plasma en la novela. (PAAU)

(41) No se trata, pues, de que el hombre vive y luego, si viene el caso, si siente alguna especial curiosidad, se ocupe en formarse algunas ideas sobre las cosas. (UNED)

\subsubsection{Las preposiciones tras, ante y hacia}

Los aprendices de ELE usan con una frecuencia inusualmente baja las preposiciones tras, ante y hacia (Cuadros 23-25).

La baja frecuencia de tras se debe a la preferencia de los aprendices por la locución preposicional equivalente después de (42), que usan con una frecuencia superior al corpus de referencia.

(42) Sin embargo, después de empezar la Reconquista, el estado de los judíos se cambiaron mal, porque los cristianos odiaban los judíos y los persiguieron en todas las partes de España. (APU)

La preposición ante aparece solo tres veces en el corpus de aprendices, una de ellas dentro de una cita, mientras que en los corpus de referencia se usa esta preposición como variante formal de delante de, combinándola frecuentemente con los sustantivos actitud, situación, postura (43-45).

(43) Sobre las tensiones políticas de agosto de 1917 puede leerse el discurso en las Cortes de Eduardo Dato, quien replica a la minoría socialista su actitud ante lo sucedido en el trágico verano y a la vez defiende la actitud del gobierno. (UNED)

(44) Nos encontramos ante una situación crítica para las formas de vida del planeta Tierra. (PAAU)

(45) Existe también un determinismo en los comportamientos humanos 
fruto de la influencia de las costumbres del grupo y, por último, un relativismo cultural que aparece al tomar una postura neutral ante la variabilidad de las culturas. (UNED)

En los corpus de referencia también se usa con más frecuencia la preposición hacia, combinada con sustantivos como camino, tendencia, respeto, actitud (47) y verbos como dirigirse $(46,48)$.

(46) En la primera parte del capítulo me ocupo de los acontecimientos del proceso histórico y educativo que considero relacionados con los principales agentes sociales hacia los que se dirige la educación intercultural: los pueblos indígenas originarios de Bolivia. (UNED)

(47) Ya tras la primera lectura se nota la actitud subjetiva, hacia el libro, del autor de este texto; una actitud muy positiva hacia aquel. (PAAU)

(48) Para cualquiera resulta obvio que la violencia que el cobrador ejerce va dirigida hacia sus deudores y víctimas, los que lo tienen todo. (UNAM)

\section{Conclusiones}

La comparación de un corpus de aprendices de ELE con varios corpus de referencia de hablantes de español como primera lengua nos ha proporcionado información sobre las palabras gramaticales más o menos frecuentes en la interlengua de los aprendices, información que esperamos pueda ser usada para desarrollar actividades didácticas encaminadas al perfeccionamiento de la destreza escrita en los niveles intermedio y avanzado.

En primer lugar, los aprendices de ELE tienen preferencia por las palabras más frecuentes en detrimento de su variante formal, como indica la alta frecuencia del determinante mисho para expresar cantidad, la conjunción causal porque (vs pues) para expresar causa, la conjunción según (vs de acuerdo con) para indicar el origen de la información, la locución después de (vs tras) para indicar orden y las preposiciones ante y hacia, que suelen complementar sustantivos abstractos como actitud, tendencia, situación, 
postura, camino, entre otros. En este sentido, se podrían diseñar ejercicios para aumentar la variedad de uso de los conectores propios del discurso académico y la riqueza de vocabulario (combinaciones de sustantivos o verbos con preposiciones).

En segundo lugar, nuestros aprendices usan con menos frecuencia de la esperada los pronombres relativos. Por lo tanto, es necesario realizar ejercicios de escritura de oraciones complejas, propias del discurso escrito.

En tercer lugar, en el corpus de aprendices y los corpus de referencia se usan algunas palabras gramaticales no solo con distinta frecuencia sino también con distintas funciones. Mientras que los autores nativos usan los demostrativos (determinantes o pronombres) como ese y aquel y pronombres personales como ello o ellos principalmente con función anafórica, para hacer referencia a entidades mencionadas con anterioridad, los aprendices o bien usan poco estas palabras o las usan mucho pero con otra función:

- Usan poco el determinante demostrativo aquel y el pronombre personal ello.

- Usan mucho el demostrativo eso pero sobre todo en la locución conjuntiva por eso.

- Usan mucho el pronombre personal ellos pero sobre todo para hacer explícito (a menudo innecesariamente) un sujeto personal.

En definitiva, para formar bien un texto es necesario hacer hincapié no solo en la formación de oraciones, sino también en los mecanismos de cohesión textual que usamos para relacionar esas oraciones formando párrafos. Entre esos mecanismos, debemos prestar especial atención al uso de pronombres demostrativos y personales.

Por último, los corpus de los que disponemos sugieren que en el discurso académico nativo es común el uso de la primera persona plural (los determinantes nuestro y los pronombre nosotros, nuestros, nos) para hacer referencia a la persona que escribe y por lo tanto hay que recordar a los aprendices que, a diferencia de la primera persona del singular, la primera persona del plural no debe evitarse. 


\section{Agradecimientos}

Esta investigación ha sido financiada por kakenhi (25770207), Grant-in-Aid for Scientific Research de la Japan Society for the Promotion of Science.

\section{Notas}

1) En la actualidad no existe un corpus representativo del español académico actual que sea de gran tamaño y que además permita no solo la consulta sino también el acceso directo a los textos. El Corpus Académico y Profesional del Español $P U C V$-2006, de 59 millones de palabras, parece no estar actualmente disponible para la consulta en web http://www.elv.cl/

2) http://biblio15.uned.es/

3) La lista completa de etiquetas usadas por Freeling y su significado puede consultarse en: http://nlp.lsi.upc.edu/freeling/doc/tagsets/tagset-es.html

4) http://www.sketchengine.co.uk/

5) Las expresiones regulares necesarias para obtener cada categoría gramatical en Sketch Engine (Freeling versión 4) son las siguientes: determinantes $\left(*^{*}-\mathrm{x}\right)$, pronombres $\left(*^{*}-\mathrm{p}\right)$, conjunciones $\left({ }^{*}-\mathrm{c}\right)$ y preposiciones $\left({ }^{*}-\mathrm{i}\right)$.

6) En los cuadros mostramos solamente los lemas con un puntaje superior a 2 (es decir, cuya frecuencia es el doble en un corpus respecto al otro), ya que un puntaje menor es poco relevante.

7) Indicamos la procedencia de los ejemplos con las siglas siguientes: APU (corpus de aprendices de ELE), UNED (corpus de tesis de la UNED), PAAU (corpus PAAU), UNAM (parte del corpus CLAE).

8) La alta frecuencia de tú, usted y vos en los cuadros 8, 9 y 10 se debe a que una de las tesinas trata sobre el uso de estos pronombres en español, es decir, se hace de ellos un uso metalingüístico.

\section{Referencias}

Battaner, P y Torner, S. (ed.), 2005. El corpus PAAU 1992: estudios descriptivos, textos y vocabulario Barcelona: Institut Universitari de Lingüística Aplicada. Universitat Pompeu Fabra; Documenta Universitaria. Disponible en http://www. iula.upf.edu/rec/corpus92/

Biber, D., S. Johansson, G. Leech, S. Conrad y Finegan, E., 1999. Longman grammar of spoken and written English, New York, Longman.

Biber, D., 2006 University Language: A corpus-based study of spoken and written registers. John 
Benjamins.

CLAE: Corpus de lenguaje académico en español. 2009. UCMexus-CONACYT. Disponible en http:// www.lenguajeacademico.info

Gallardo, S. (2005), La monografía universitaria como aprendizaje para la producción de artículos científicos. En Vázquez, G. (Coord.), Español con fines académicos: de la comprensión a la producción de textos, Edinumen.

Padró, L1., Stanilovsky, E., 2012. FreeLing 3.0: Towards Wider Multilinguality Proceedings of the Language Resources and Evaluation Conference (LREC 2012) ELRA. Istanbul, Turkey. May, 2012.

Paquot, M., (2010). Academic Vocabulary in Learner Writing. Continuum (2010)

Kilgarriff, A., 2009. Simple maths for keywords. En Mahlberg, M., González-Díaz, V. y Smith, C. (eds.) Proceedings of the Corpus Linguistics Conference CL2009. University of Liverpool, UK.

Kilgarrif, A., 2012. "Getting to know your corpus". Proceedings of Text, Speech and Dialogue 2012, Lecture Notes in Computer Science. Springer.

Kilgarrif, A., 2013. Terminology finding, parallel corpora and bilingual word sketches in the Sketch Engine ASLIB 35th Translating and the Computer conference, London.

Kilgarriff, Adam, et al. (2014). The Sketch Engine: ten years on. En Lexicography (2014): 1-30.

Valverde, M.P., 2011. An Evaluation of Part of Speech Tagging on Written Second Language Spanish. Gelbukh, Alexander (Ed.), Lecture Notes in Computer Science, vol. 6609, SpringerLink, pp. 214-226. 


\section{Apéndice: Cuadros.}

En los cuadros mostramos solamente los lemas con un puntaje superior a 2 (es decir, cuya frecuencia es el doble en un corpus respecto al otro), ya que un puntaje menor es poco relevante.

Cuadro 1:Análisis morfológico de una frase del corpus de aprendices

\begin{tabular}{l|ll|} 
Forma & Lema & Categoria gr. \\
\hline Especialmente & especialmente & RG \\
\hline en & en & SPS00 \\
\hline España & españa & NP00000 \\
\hline y & y & CC \\
Italia & italia & NP00000 \\
\hline se & se & POOCNO00 \\
\hline cultiva & cultivar & VMIP3SO \\
\hline bastante & bastante & RG \\
\hline
\end{tabular}

Cuadro 2:Determinantes usados con más frecuencia en el corpus de aprendices respecto al corpus UNED:No consideramos significativa la frecuencia de qué debido a que su repetición se concentra principalmente en solo uno de los resúmenes:

\begin{tabular}{lrrrrr}
\multicolumn{4}{c}{ APU } & \multicolumn{2}{c}{ UNED } \\
\hline \multicolumn{1}{c}{ lempos } & Freq & Freq/mill (?) & Freq & Freq/mill & Score \\
\hline mucho-x & $\underline{147}$ & 1988.8 & $\underline{1.271}$ & 351.3 & 4.6 \\
qué-x & $\underline{\mathbf{2 6}}$ & 351.8 & $\underline{402}$ & 111.1 & 2.1 \\
varios-x & $\underline{\mathbf{3 8}}$ & 514.1 & $\underline{\mathbf{7 4 4}}$ & 205.7 & 2.0
\end{tabular}

Cuadro 3:Determinantes usados con más frecuencia en el corpus de aprendices respecto al corpus PAAU.

\begin{tabular}{lrrrrr} 
& \multicolumn{2}{c}{ APU } & \multicolumn{4}{c}{ PAAU } \\
\hline \multicolumn{1}{c}{ lempos } & Freq & Freq/mill ? & Freq & Freq/mill & Score \\
\hline mucho-x & $\underline{147}$ & 1988.8 & $\underline{264}$ & 601.3 & 3.0 \\
qué-x & $\underline{26}$ & 351.8 & $\underline{25}$ & 56.9 & 2.9
\end{tabular}


Cuadro 4:Determinantes usados con más frecuencia en el corpus de aprendices respecto al corpus UNAM.

\begin{tabular}{crrrrr}
\multicolumn{4}{c}{ APU } & \multicolumn{2}{c}{ UNAM } \\
\hline lempos & Freq & Freq/mill ? & Freq & Freq/mill & Score \\
\hline mucho-x & $\underline{147}$ & 1988.8 & $\underline{232}$ & 787.1 & 2.4
\end{tabular}

Cuadro 5:Determinantes usados con menos frecuencia en el corpus de aprendices respecto al corpus de tesis UNED.

\begin{tabular}{lcrrrr}
\multicolumn{2}{c}{ UNED } & \multicolumn{2}{c}{ APU } \\
\hline \multicolumn{1}{c}{ lempos } & Freq & Freq/mill ? & Freq & Freq/mill & Score \\
\hline aquel-x & $\underline{1,071}$ & 296.1 & $\underline{2}$ & 27.1 & 3.1 \\
nuestro-x & $\underline{2.689}$ & 743.3 & $\underline{19}$ & 257.1 & 2.4
\end{tabular}

Cuadro 6:Determinantes usados con menos frecuencia en el corpus de aprendices respecto al corpus PAAU.

\begin{tabular}{lrrrrr} 
& \multicolumn{2}{c}{ PAAU } & \multicolumn{2}{c}{ APU } \\
\hline \multicolumn{1}{c}{ lempos } & Freq & Freq/mill ? & Freq & Freq/mill & Score \\
\hline aquel-x & $\underline{139}$ & 316.6 & $\underline{2}$ & 27.1 & 3.3 \\
todo-X & $\underline{1,186}$ & 2701.2 & $\underline{82}$ & 1109.4 & 2.3 \\
ojo-X & $\underline{50}$ & 113.9 & 0 & 0.0 & 2.1 \\
uno-x & $\underline{8.710}$ & 19837.8 & $\underline{695}$ & 9402.7 & 2.1 \\
nuestro-x & $\underline{\mathbf{2 7 8}}$ & 633.2 & $\underline{19}$ & 257.1 & 2.1
\end{tabular}

Cuadro 7:Determinantes usados con menos frecuencia en el corpus de aprendices respecto al corpus UNAM.

\begin{tabular}{lrrrrr} 
& \multicolumn{2}{c}{ UNAM } & \multicolumn{2}{c}{ APU } \\
\hline \multicolumn{1}{c}{ lempos } & Freq & Freq/mill ? & Freq & Freq/mill & Score \\
\hline aquel-x & $\underline{118}$ & 400.3 & $\underline{2}$ & 27.1 & 3.9 \\
nuestro-x & $\underline{297}$ & 1007.6 & $\underline{19}$ & 257.1 & 3.1 \\
tal-x & $\underline{191}$ & 648.0 & $\underline{14}$ & 189.4 & 2.6 \\
\hline
\end{tabular}

Cuadro 8:Pronombres usados con más frecuencia en el corpus de aprendices respecto al corpus de tesis UNED.

\begin{tabular}{lrrrrr} 
& \multicolumn{4}{c}{ APU } & \multicolumn{4}{c}{ UNED } \\
\hline lempos & Freq & Freq/mill ? & \multicolumn{1}{c}{ Freq } & Freq/mill & Score \\
\hline tú-p & $\underline{70}$ & 947.0 & $\underline{44}$ & 12.2 & 9.3 \\
ese-p & $\underline{177}$ & 2394.6 & $\underline{804}$ & 222.3 & 7.7 \\
usted-p & $\underline{46}$ & 622.3 & $\underline{36}$ & 10.0 & 6.6 \\
ellos-p & $\underline{182}$ & 2462.3 & $\underline{2.266}$ & 626.4 & 3.5 \\
vos-p & $\underline{10}$ & 135.3 & $\underline{52}$ & 14.4 & 2.1 \\
\hline
\end{tabular}


Cuadro 9:Pronombres usados con más frecuencia en el corpus de aprendices respecto al corpus PAAU.

\begin{tabular}{lrrrrr} 
& \multicolumn{4}{c}{ APU } & \multicolumn{2}{c}{ PAAU } \\
\hline lempos & Freq & Freq/mill ? & Freq & Freq/mill & Score \\
\hline tú-p & $\underline{70}$ & 947.0 & $\underline{7}$ & 15.9 & 9.0 \\
usted-p & $\underline{46}$ & 622.3 & $\underline{1}$ & 2.3 & 7.1 \\
ese-p & $\underline{177}$ & 2394.6 & $\underline{135}$ & 307.5 & 6.1 \\
cómo-p & $\underline{38}$ & 514.1 & $\underline{40}$ & 91.1 & 3.2 \\
ellos-p & $\underline{182}$ & 2462.3 & $\underline{363}$ & 826.8 & 2.8 \\
vos-p & $\underline{10}$ & 135.3 & $\underline{3}$ & 6.8 & 2.2 \\
qué-p & $\underline{28}$ & 378.8 & $\underline{52}$ & 118.4 & 2.2
\end{tabular}

Cuadro 10:Pronombres usados con más frecuencia en el corpus de aprendices respecto al corpus UNAM.

\begin{tabular}{lrrrrr} 
& \multicolumn{2}{c}{ APU } & \multicolumn{2}{c}{ UNAM } \\
\hline lempos & Freq & Freq/mill ? & Freq & Freq/mill & Score \\
\hline tú-p & $\underline{70}$ & 947.0 & $\underline{5}$ & 17.0 & 9.0 \\
ese-p & $\underline{177}$ & 2394.6 & $\underline{98}$ & 332.5 & 5.8 \\
usted-p & $\underline{46}$ & 622.3 & $\underline{29}$ & 98.4 & 3.6 \\
ellos-p & $\underline{182}$ & 2462.3 & $\underline{204}$ & 692.1 & 3.2
\end{tabular}

Cuadro 11:Pronombres usados con menos frecuencia en el corpus de aprendices respecto al corpus de tesis UNED.

\begin{tabular}{llrrrr} 
& \multicolumn{2}{c}{ UNED } & \multicolumn{3}{c}{ APU } \\
\hline \multicolumn{1}{c}{ lempos } & Freq & Freq/mill ? & Freq & Freq/mill & Score \\
\hline cual-p & $\underline{1,879}$ & 519.4 & $\underline{5}$ & 67.6 & 3.7 \\
nos-p & $\underline{\underline{2,544}}$ & 703.2 & $\underline{10}$ & 135.3 & 3.4 \\
ello-p & $\underline{1,576}$ & 435.7 & $\underline{5}$ & 67.6 & 3.2 \\
donde-p & $\underline{1,917}$ & 529.9 & $\underline{12}$ & 162.3 & 2.4 \\
cuyo-p & $\underline{1,097}$ & 303.2 & $\underline{6}$ & 81.2 & 2.2 \\
tal-p & $\underline{547}$ & 151.2 & $\underline{1}$ & 13.5 & 2.2 \\
aquel-p & $\underline{401}$ & 110.8 & 0 & 0.0 & 2.1
\end{tabular}

Cuadro 12:Pronombres usados con menos frecuencia en el corpus de aprendices respecto al corpus PAAU.

\begin{tabular}{lrrrrr}
\multicolumn{1}{c}{ PAAU } & \multicolumn{3}{c}{ APU } \\
\hline \multicolumn{1}{c}{ lempos } & Freq & Freq/mill ? & Freq & Freq/mill & Score \\
\hline nos-p & $\underline{877}$ & 1997.4 & $\underline{10}$ & 135.3 & 8.9 \\
cual-p & $\underline{514}$ & 1170.7 & $\underline{5}$ & 67.6 & 7.6 \\
donde-p & $\underline{433}$ & 986.2 & $\underline{12}$ & 162.3 & 4.1 \\
ello-p & $\underline{243}$ & 553.5 & $\underline{5}$ & 67.6 & 3.9 \\
aquel-p & $\underline{89}$ & 202.7 & $\underline{0}$ & 0.0 & 3.0 \\
algo-p & $\underline{189}$ & 430.5 & $\underline{7}$ & 94.7 & 2.7 \\
este-p & $\underline{1,052}$ & 2396.0 & $\underline{68}$ & 920.0 & 2.4 \\
nosotros-p & $\underline{94}$ & 214.1 & $\underline{3}$ & 40.6 & 2.2 \\
le-p & $\underline{597}$ & 1359.7 & $\underline{42}$ & 568.2 & 2.2 \\
demás-p & $\underline{\mathbf{7 6}}$ & 173.1 & $\underline{2}$ & 27.1 & 2.1 \\
cuyo-p & $\underline{122}$ & 277.9 & $\underline{6}$ & 81.2 & 2.1
\end{tabular}


Cuadro 13:Pronombres usados con menos frecuencia en el corpus de aprendices respecto al corpus UNAM.

\begin{tabular}{lrrrrr} 
& \multicolumn{3}{c}{ UNAM } & \multicolumn{3}{c}{ APU } \\
\hline \multicolumn{1}{c}{ lempos } & Freq & Freq/mill ? & Freq & Freq/mill & Score \\
\hline cual-p & $\underline{316}$ & 1072.1 & $\underline{5}$ & 67.6 & 7.0 \\
nos-p & $\underline{\underline{383}}$ & 1299.4 & $\underline{10}$ & 135.3 & 5.9 \\
donde-p & $\underline{247}$ & 838.0 & $\underline{12}$ & 162.3 & 3.6 \\
quien-p & $\underline{177}$ & 600.5 & $\underline{10}$ & 135.3 & 3.0 \\
ello-p & $\underline{117}$ & 396.9 & $\underline{5}$ & 67.6 & 3.0 \\
le-p & $\underline{500}$ & 1696.3 & $\underline{42}$ & 568.2 & 2.7 \\
aquel-p & $\underline{45}$ & 152.7 & 0 & 0.0 & 2.5 \\
quién-p & $\underline{44}$ & 149.3 & 0 & 0.0 & 2.5 \\
me-p & $\underline{162}$ & 549.6 & $\underline{12}$ & 162.3 & 2.5 \\
cuyo-p & $\underline{96}$ & 325.7 & $\underline{6}$ & 81.2 & 2.3 \\
algo-p & $\underline{103}$ & 349.4 & $\underline{7}$ & 94.7 & 2.3 \\
tal-p & $\underline{40}$ & 135.7 & $\underline{1}$ & 13.5 & 2.1
\end{tabular}

Cuadro 14:Conjunciones usadas con más frecuencia en el corpus de aprendices respecto al corpus de tesis UNED.

\begin{tabular}{crrrrr}
\multicolumn{2}{c}{ APU } & \multicolumn{2}{c}{ UNED } \\
\hline lempos & Freq & Freq/mill ? & Freq & Freq/mill & Score \\
\hline porque-c & $\underline{139}$ & 1880.5 & $\underline{1,858}$ & 513.6 & 3.2
\end{tabular}

Cuadro 15:Conjunciones usadas con más frecuencia en el corpus de aprendices respecto al corpus PAAU.

\begin{tabular}{crrrrr} 
& \multicolumn{2}{c}{ APU } & \multicolumn{2}{c}{ PAAU } \\
\hline lempos & Freq & Freq/mill ? & Freq & Freq/mill & Score \\
\hline porque-c & $\underline{139}$ & 1880.5 & $\underline{382}$ & 870.0 & 2.0
\end{tabular}

Cuadro 16:Conjunciones usadas con más frecuencia en el corpus de aprendices respecto al corpus UNAM.

\begin{tabular}{crrrrr}
\multicolumn{2}{c}{ APU } & \multicolumn{2}{c}{ UNAM } \\
\hline lempos & Freq & Freq/mill ? & Freq & Freq/mill & Score \\
\hline porque-c & $\underline{139}$ & 1880.5 & $\underline{209}$ & 709.1 & 2.4
\end{tabular}

Cuadro 17:Conjunciones usadas con menos frecuencia en el corpus de aprendices respecto al corpus de tesis UNED.

\begin{tabular}{clrrrr}
\multicolumn{2}{c}{ UNED } & \multicolumn{4}{c}{ APU } \\
\hline lempos & Freq & Freq/mill ? & Freq & Freq/mill & Score \\
\hline pues-c & $\underline{2.245}$ & 620.6 & $\underline{16}$ & 216.5 & 2.3
\end{tabular}


Cuadro 18:Conjunciones usadas con menos frecuencia en el corpus de aprendices respecto al corpus PAAU.

\begin{tabular}{lrrrrr} 
& \multicolumn{2}{c}{ PAAU } & \multicolumn{2}{c}{ APU } \\
\hline lempos & Freq & Freq/mill ? & Freq & Freq/mill & Score \\
\hline mas-c & $\underline{118}$ & 268.8 & $\underline{2}$ & 27.1 & 2.9 \\
pues-c & $\underline{274}$ & 624.1 & $\underline{16}$ & 216.5 & 2.3
\end{tabular}

Cuadro 19:Conjunciones usadas con menos frecuencia en el corpus de aprendices respecto al corpus UNAM.

\begin{tabular}{lrrrrr} 
& \multicolumn{2}{c}{ UNAM } & \multicolumn{2}{c}{ APU } \\
\hline \multicolumn{1}{c}{ lempos } & Freq & Freq/mill ? & Freq & Freq/mill & Score \\
\hline mas-c & $\underline{104}$ & 352.8 & $\underline{2}$ & 27.1 & 3.6 \\
pues-c & $\underline{249}$ & 844.8 & $\underline{16}$ & 216.5 & 3.0
\end{tabular}

Cuadro 20:Preposiciones usadas con más frecuencia en el corpus de aprendices respecto al corpus de tesis UNED.

\begin{tabular}{crrrrr}
\multicolumn{2}{c}{ APU } & \multicolumn{2}{c}{ UNED } \\
\hline lempos & Freq & Freq/mill ? & Freq & Freq/mill & Score \\
\hline según-i & $\underline{82}$ & 1109.4 & $\underline{1,617}$ & 447.0 & 2.2
\end{tabular}

Cuadro 21:Preposiciones usadas con más frecuencia en el corpus de aprendices respecto al corpus PAAU.

\begin{tabular}{lrrrrr} 
& \multicolumn{2}{c}{ APU } & \multicolumn{2}{c}{ PAAU } \\
\hline lempos & Freq & Freq/mill ? & Freq & Freq/mill & Score \\
\hline desde-1 & $\underline{132}$ & 1785.8 & $\underline{237}$ & 539.8 & 2.9 \\
según-1 & $\underline{82}$ & 1109.4 & $\underline{216}$ & 492.0 & 2.0
\end{tabular}

Cuadro 22:Preposiciones usadas con más frecuencia en el corpus de aprendices respecto al corpus UNAM.

\begin{tabular}{lrrrrr}
\multicolumn{4}{c}{ APU } & \multicolumn{4}{c}{ UNAM } \\
\hline lempos & Freq & Freq/mill ? & Freq & Freq/mill & Score \\
\hline contra-i & $\underline{40}$ & 541.2 & $\underline{43}$ & 145.9 & 2.6 \\
según-1 & $\underline{82}$ & 1109.4 & $\underline{110}$ & 373.2 & 2.6
\end{tabular}

Cuadro 23:Preposiciones usadas con menos frecuencia en el corpus de aprendices respecto al corpus de tesis UNED.

\begin{tabular}{lcrrrr} 
& \multicolumn{2}{c}{ UNED } & \multicolumn{2}{c}{ APU } \\
\hline \multicolumn{1}{c}{ lempos } & Freq & Freq/mill ? & Freq & Freq/mill & Score \\
\hline ante-1 & $\underline{1,353}$ & 374.0 & $\underline{3}$ & 40.6 & 3.4 \\
tras-i & $\underline{949}$ & 262.3 & $\underline{1}$ & 13.5 & 3.2
\end{tabular}


Cuadro 24:Preposiciones usadas con menos frecuencia en el corpus de aprendices respecto al corpus PAAU.

\begin{tabular}{lrrrrr}
\multicolumn{2}{c}{ PAAU } & \multicolumn{3}{c}{ APU } \\
\hline \multicolumn{1}{c}{ lempos } & Freq & Freq/mill ? & Freq & Freq/mill & Score \\
\hline ante-1 & $\underline{218}$ & 496.5 & $\underline{3}$ & 40.6 & 4.2 \\
tras-1 & $\underline{130}$ & 296.1 & $\underline{1}$ & 13.5 & 3.5 \\
hacia-i & $\underline{246}$ & 560.3 & $\underline{11}$ & 148.8 & 2.7 \\
mediante-i & $\underline{179}$ & 407.7 & $\underline{\mathbf{Z}}$ & 94.7 & 2.6
\end{tabular}

Cuadro 25:Preposiciones usadas con menos frecuencia en el corpus de aprendices respecto al corpus UNAM.

\begin{tabular}{crrrrr} 
& \multicolumn{2}{c}{ UNAM } & \multicolumn{3}{c}{ APU } \\
\hline lempos & Freq & Freq/mill ? & \multicolumn{1}{c}{ Freq } & Freq/mill & Score \\
\hline ante-1 & $\underline{90}$ & 305.3 & $\underline{3}$ & 40.6 & 2.9
\end{tabular}


$<$ Resumen $>$

\title{
Frecuencia de uso de palabras gramaticales en textos académicos:
}

\author{
Comparación de un corpus de aprendices \\ de ELE con tres corpus de referencia
}

\begin{abstract}
María del Pilar VALVERDE IBÁÑEZ
Los textos escritos por aprendices de ELE se diferencian de los textos escritos por hablantes nativos no solo por el hecho de contener mayor número de errores gramaticales o léxicos, sino también, especialmente en los niveles intermedio-alto y avanzado, por la mayor o menor frecuencia de ciertas palabras y estructuras. La presente investigación trata sobre la frecuencia de uso de las palabras gramaticales (determinantes, pronombres, conjunciones y preposiciones) en un corpus de textos académicos (resúmenes de tesinas de graduación) producidos por universitarios japoneses que estudian español como lengua extranjera, en comparación con tres corpus académicos de referencia. Analizamos con detalle los contextos de aparición de las palabras más o menos frecuentes en un corpus respecto al otro y extraemos algunas conclusiones sobre las estrategias de producción de textos escritos a las que los aprendices necesitan prester más atención (principalmente la variedad léxica, el uso de oraciones subordinadas y los mecanismos de cohesión).
\end{abstract}

